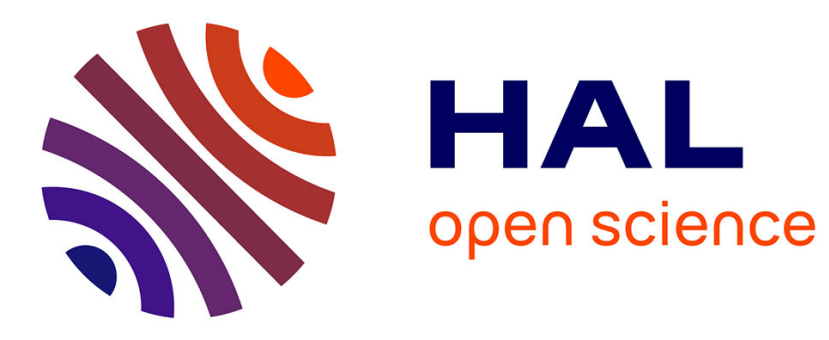

\title{
Using a plasmonic lens to control the emission of electrically excited light
}

Shuiyan Cao, Médéric Lequeux, Eric Le Moal, Aurélien Drezet, Serge Huant, Gérald Dujardin, Elizabeth Boer-Duchemin

\section{- To cite this version:}

Shuiyan Cao, Médéric Lequeux, Eric Le Moal, Aurélien Drezet, Serge Huant, et al.. Using a plasmonic lens to control the emission of electrically excited light. Proceedings of SPIE, the International Society for Optical Engineering, 2016, Nanophotonics VI, 9884, pp.98841Y. 10.1117/12.2224472 . hal-01449022

\section{HAL Id: hal-01449022 \\ https://hal.science/hal-01449022}

Submitted on 29 Jan 2017

HAL is a multi-disciplinary open access archive for the deposit and dissemination of scientific research documents, whether they are published or not. The documents may come from teaching and research institutions in France or abroad, or from public or private research centers.
L'archive ouverte pluridisciplinaire HAL, est destinée au dépôt et à la diffusion de documents scientifiques de niveau recherche, publiés ou non, émanant des établissements d'enseignement et de recherche français ou étrangers, des laboratoires publics ou privés. 


\title{
Using a plasmonic lens to control the emission of electrically excited light
}

\author{
Shuiyan $\mathrm{Cao}^{a}$, Médéric Lequeux ${ }^{a}$, Eric Le Moal ${ }^{a}$, Aurélien Drezet $^{b}$, Serge Huant ${ }^{b}$, Gérald \\ Dujardin $^{a}$, Elizabeth Boer-Duchemin ${ }^{a}$ \\ ${ }^{a}$ Institut des Sciences Moléculaires d'Orsay (ISMO), CNRS, Univ Paris Sud, Université \\ Paris-Saclay, F-91405 Orsay (France); \\ ${ }^{b}$ Université Grenoble Alpes, Institut NEEL, F-38000 Grenoble, France and CNRS, Institut \\ NEEL, F-38042 Grenoble, France.
}

\begin{abstract}
A local, low-energy, electrical method for the excitation of localized and propagating surface plasmon polaritons (SPPs) is attractive for both fundamental and applied research. In particular, such a method produces no excitation background light and may be integrated with nanoelectronics. Here we report on the electrical excitation of SPPs through the inelastic tunneling of low-energy electrons from the tip of a scanning tunneling microscope (STM) to the surface of a two-dimensional plasmonic lens. The plasmonic structure is a series of concentric circular slits etched in a thick gold film on a glass substrate. An out-going circular SPP wave is generated from the tip-sample junction and is scattered into light by the slits. We compare the resulting emission pattern to that observed when exciting SPPs on a thin, unstructured gold film. For optimized parameters, the light emitted from the plasmonic lens is radially polarized. We describe the effects of the slit period and number, and lens diameter on the emission pattern and we diskuss how the light beam of low divergence is formed.
\end{abstract}

Keywords: Surface plasmon, scanning tunneling microscopy, plasmonic lens, inelastic electron tunneling, light nanosource

\section{INTRODUCTION}

Surface plasmon polaritions (SPPs) have elicited considerable attention during the last decade with applications ranging from high-density optical data storage ${ }^{1,2}$ to light focusing. ${ }^{3}$ Plasmons may even one day replace electrons or photons for data transfer and computation and thus overcome the disadvantages of the lower speed of electronics and the larger size of photonic devices. ${ }^{4}$ Up to now, many different ways of exciting SPPs have been reported. ${ }^{5}$ More recently, a scanning tunneling microscope (STM) has been used to excite both localized and propagating SPPs. ${ }^{6,7}$ This method is preferable due to its advantages such as the low energy that is needed and the fact that no excitation background light is introduced. ${ }^{8,9}$ As well, the extreme spatial selectivity means that a specific mode of a plasmonic structure may be excited, thus leading to the control of the resulting emission of SPPs and light. ${ }^{10-13}$

In this article we report on the controllable, local, low-energy, electric excitation of SPPs achieved with tunnel electrons from the tip of an STM on a two-dimensional plasmonic lens structure. Our plasmonic lens consists of several concentric slits etched in a gold film that has a thickness of $200 \mathrm{~nm}$. With our setup, the nanosource of both localized and propagating surface plasmons is coupled to an inverted optical microscope, enabling the photon emission to be mapped in both real and Fourier spaces.

In the first part, we compare the experimental results of the STM excitation of a plasmonic lens ${ }^{14}$ with the STM excitation of a simple gold film with a thickness of $50 \mathrm{~nm}$. The plasmonic lenses we use here have five concentric slits with a period of 700 or $800 \mathrm{~nm}$ and an inner diameter of the central disk of $5 \mu \mathrm{m}$. Results in both the real and Fourier spaces show the difference between the leakage radiation from thin gold film and the

Further author information: (Send correspondence to E.L.M.)

S.C.: E-mail: shuiyan.cao@u-psud.fr, Telephone: +33 (0)169156550

E.L.M.: E-mail: eric.le-moal@u-psud.fr, Telephone: +33 (0)169156697

Nanophotonics VI, edited by David L. Andrews, Jean-Michel Nunzi, Andreas Ostendorf, Proc. of SPIE Vol. 9884, 98841Y · C 2016 SPIE · CCC code: 0277-786X/16/\$18 · doi: 10.1117/12.2224472 

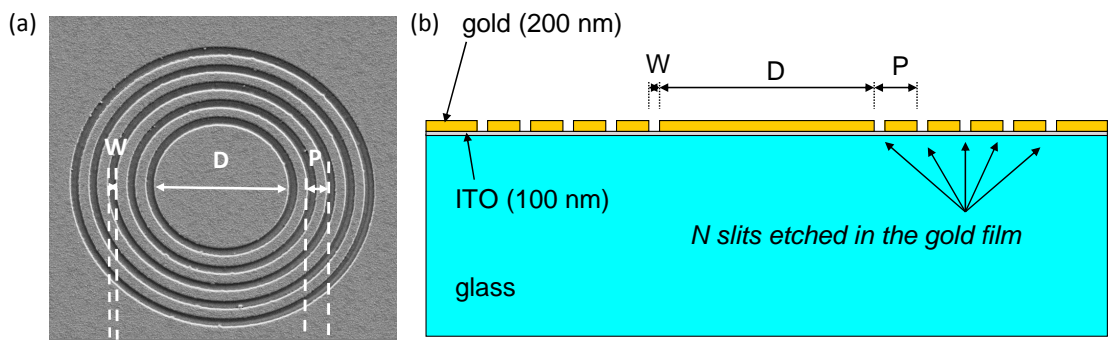

Figure 1. Plasmonic structures. (a) Scanning electron microscopy (SEM) image of a plasmonic lens. The inner diameter $D$ is $5 \mu \mathrm{m}$, the slit grating period $P$ is $700 \mathrm{~nm}$ and the slit width $W$ is $300 \mathrm{~nm}$. (b) Schematics of a plasmonic lens (transverse view). A 200-nm gold layer is evaporated on a 100-nm ITO layer on a glass substrate and etched with circular slits.

scattered light from the slits of the plasmonic lens. The leakage radiation from a thin gold film and the scattered light from a plasmonic lens are both radially polarized. Yet, the divergence angle of the emitted light from the plasmonic lens with five slits is very small compared to that of the gold film, thus leading to a well-defined beam of light.

In the second part, we will focus on excitation results for plasmonic lenses with different parameters. There are several structures with a different number of slits $(N=1,3$ and 5$)$, with different inner diameters $D$ varying from $1 \mu \mathrm{m}$ to $6 \mu \mathrm{m}$ and with different periods $(P=600,700$ and $800 \mathrm{~nm})$. Our experimental results indicate that the structure with the most number of slits has the smallest divergence angle. We attribute the low divergence to the constructive interference of the light from the multiple slits of the structure. As well, we provide a rationale for the best trade-off between the lateral size of the plasmonic lens and the collimation of the emitted light beam, in terms of angular divergence and side-lobe occurrence. Finally, we diskuss the robustness of this electrically driven light beam microsource to the off-centering of the local SPP nanosource.

\section{EXPERIMENTAL METHODS}

Figure 1 shows a scanning electron microscopy (SEM) image of one of our plasmonic lenses as well as a schematic diagram. Several concentric slits (number of slits $N$ is 1, 3 or 5) are etched in a gold film of thickness $200 \mathrm{~nm}$. A dual beam FIB (focused ion beam) and SEM system is used for the preparation of the plasmonic lens. Slit widths are $W=300 \mathrm{~nm}$ and the inner diameter of the plasmonic lens $D$ varies from 1 to $6 \mu \mathrm{m}$ in steps of $1 \mu \mathrm{m}$. Our structures have slit grating periods of $600 \mathrm{~nm}, 700 \mathrm{~nm}$ or $800 \mathrm{~nm}$. For comparison purposes, we also use a sample consisting of a continuous gold film, $50-\mathrm{nm}$ in thickness, thermally evaporated in vacuum on a glass coverslip after deposition of $2 \mathrm{~nm}$ of chromium.

Figure 2 shows our experimental set-up. An STM head with a tungsten tip is mounted above our sample. Below is an inverted optical microscopy with an oil-immersion objective lens of high numerical aperture. The STM tip is grounded and our sample is biased to $2.8 \mathrm{~V}$. Additionally, tunnel current setpoint values are in the range of $1 \mathrm{nA}$ to $6 \mathrm{nA}$. Figure 2(a) shows the configuration used for real-space and spectrum measurements. When the emitted light is focused directly on the cooled CCD camera we get a real-space image, which provides the spatial distribution of the emitted light. When the light is diverted to the spectrometer, we obtain the energy distribution of the emitted light. If we add an extra lens, the CCD camera records a Fourier space image and this is shown in Fig.2 (b). From Fourier space images, we obtain information on the angular distribution of the light emission.

\section{RESULTS AND DISKUSSION}

\subsection{Comparison between Emission Patterns on a Thin Gold Film and a Plasmonic Lens}

First, we compare the spatial and angular distribution of the light generated through the local electrical excitation of a 5-slit circular grating etched in a thick gold film (200-nm thick) and that of a thin, planar, gold film (50-nm thick). Electrical excitation is achieved using the tunnel current from the STM tip. Figure 3 shows the resulting 
(a)

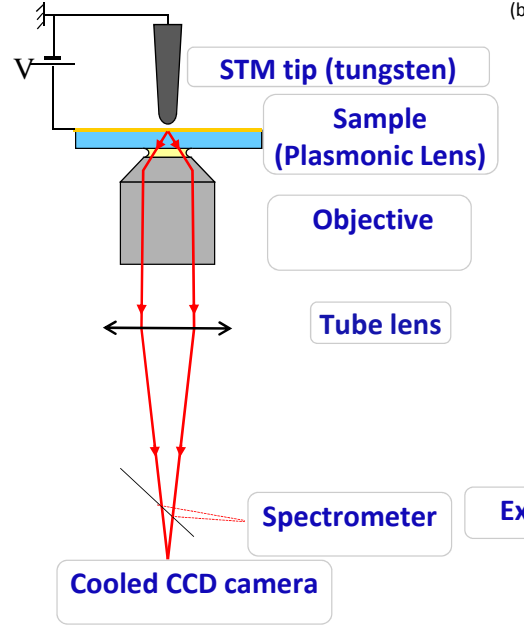

(b)

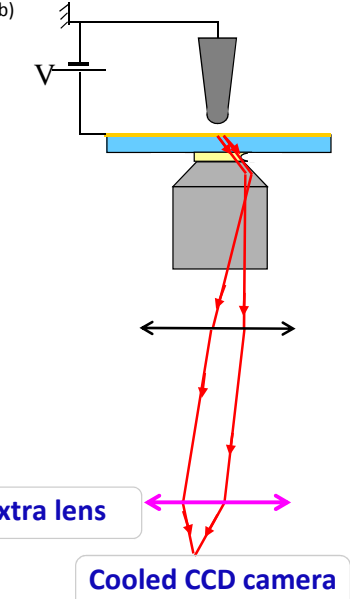

Figure 2. Experimental setup: a grounded STM head with a tungsten tip is mounted above our plasmonic structure sample which is biased and below is an inverted optical microscopy with an oil-immersion objective lens of high numerical aperture. (a) Real space and spectrum measurements. For the real space measurement, the emitted light is focused on the cooled CCD camera. For the spectrum measurement, the light is diverted to a spectrometer coupled to another CCD camera. (b) Fourier space measurement: an extra lens is added in front of the CCD camera so that Fourier plane is imaged.

real-space (a-d) and Fourier-space (e-h) images, recorded without (a,b,e,f) and with (c,d,g,h) a linear polarizer in front of the CCD camera. Schematics above the experimental images illustrate the two kinds of excited structures and the tip location. The plasmonic lens has grating period $P=700 \mathrm{~nm}$ and inner diameter $D=5 \mu \mathrm{m}$. Due to the two different gold film thicknesses, only radiative scattering of SPPs at the slits is detected in the case of the plasmonic lens, whereas only SPP leakage radiation ${ }^{15}$ is observed in the case of the thin, planar, gold film.

In the real-space images, we see that the light from the plasmonic lens is emitted from the five slits, with a much stronger contribution from the inner slit. This is expected since the SPPs scattered at the slits are a $2 \mathrm{D}$ circular out-going wave that isotropically propagates away from the center of the structure. Moreover, the SPP intensity decays very quickly when SPPs propagate through the grating (decay length $\approx 3 \mathrm{~nm}$ ). On the unstructured gold film, a 2D circular out-going wave also propagates away from the tip location, yet with a comparatively much longer propagation length $(\approx 15 \mathrm{~nm})$. High intensity is observed from below the tip, due to the excitation of localized surface plasmons that radiatively decay in the glass substrate.

In the Fourier-space images we see that the light from the plasmonic lens is essentially emitted orthogonally to the surface plane with a remarkably narrow angle of dispersion (half-width at half-maximum of $\approx 4^{\circ}$ ). Conversely, a wide emission cone is observed for the excitation of SPPs on a thin, planar, gold film, with the emission maximum at the polar angle corresponding to SPP-light resonant coupling, i.e., at about $43^{\circ}$. Light at emission angles up to the maximum acceptance of the objective lens is recorded.

A clear symmetry appears between the image series on the left and right sides of Fig. 3. Real-space images on the thin, planar, gold film exhibits a sharp doughnut shaped spot, like the Fourier-space images of the plasmonic 

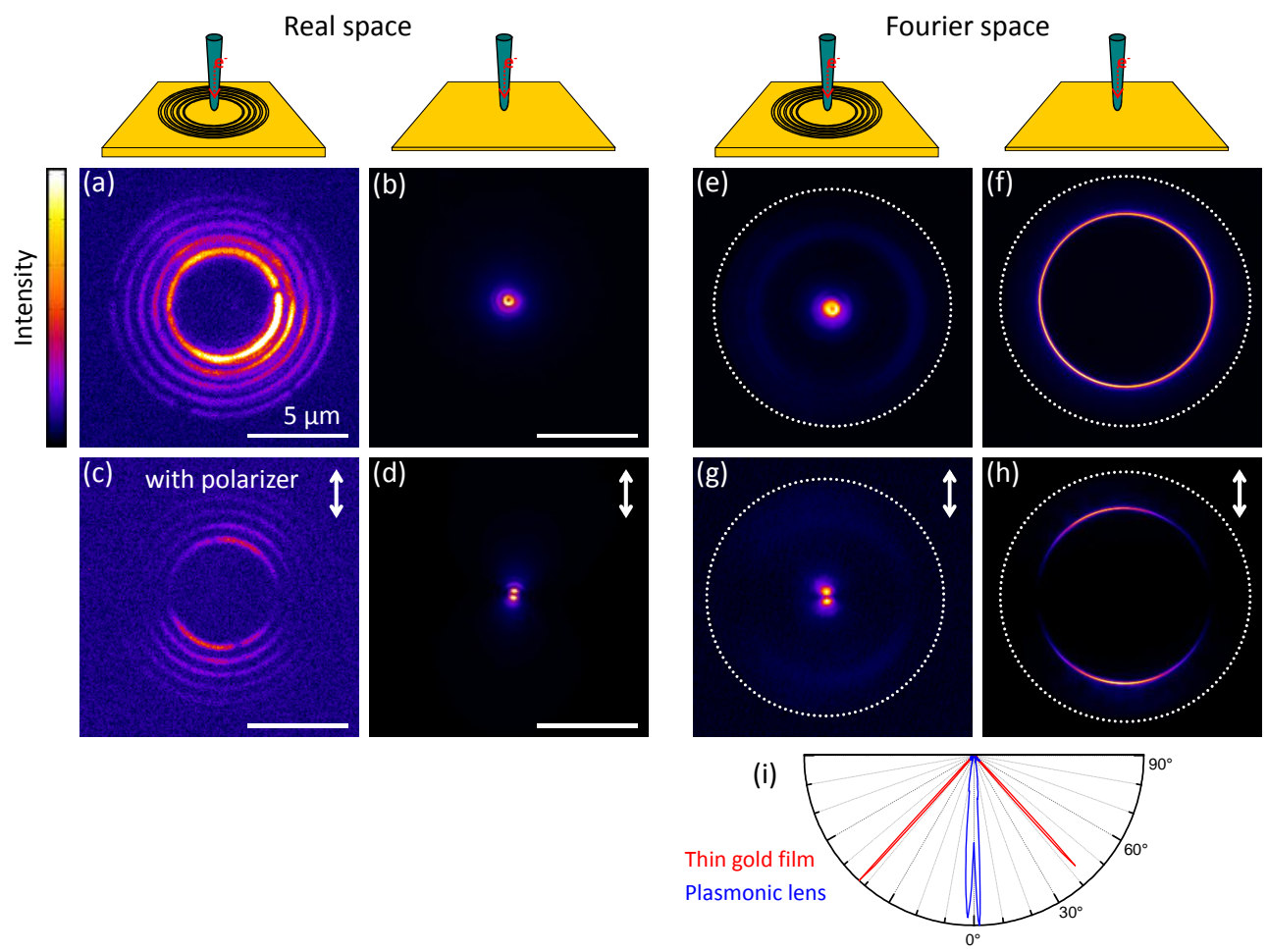

Figure 3. Comparison of the light emitted from a plasmonic lens and a thin gold film after STM plasmon excitation. (a) to (d) Real-space images in false color (a,c) from a plasmonic lens and (b,d) from a thin gold film, (a,b) without and (c,d) with a linear polarizer in front of the detector. (e) to (h) Fourier-space optical images in false color (e,g) from a plasmonic lens and $(\mathrm{e}, \mathrm{g})$ from a $50-\mathrm{nm}$ thin gold film, $(\mathrm{e}, \mathrm{f})$ without and $(\mathrm{g}, \mathrm{h})$ with a linear polarizer in front of the detector. The white arrow indicates the polarization axis. (i) Polar plots of the angular emission pattern from a plasmonic lens (blue line) and a thin gold film (red line). The half-width-half-maximum of the beam from the plasmonic lens is about $4^{\circ}$, and the angle of maximum emission for the thin film is $\approx 43^{\circ}$. 
lens. Emission from the plasmonic lens is spatially delocalized and angularly narrow, whereas emission from the unstructured gold film is partly localized below the tip and strongly diverges. In all images, when a polarizer is placed in front of the detector, the intensity cancels out along the axis orthogonal to the polarizer transmission axis. This denotes radially polarized (or $p$-polarized) emission.

If is of interest to compare these two sources of light in terms of how "spread out" their emission is in area and angle. In the case of the plasmonic lens (see Fig. 1(a)), light is exclusively emitted from the slits etched in the thick gold film. The spatial footprint of the source in the plane is thus limited to a disk whose diameter is the outer diameter $D^{\prime}$ of the plasmonic lens. The structure shown in Fig. 1(a) has $D^{\prime}=11.2 \mu \mathrm{m}$, which yields a footprint $\Sigma=\frac{\pi}{4} D^{\prime 2} \approx 100 \mu \mathrm{m}^{2}$. In the case of the thin continuous gold film (see Fig. 1(b)), the spatial footprint of the source is more difficult to define, because SPPs propagates away from the tip location, along the air-gold interface, until their energy is dissipated in the metal or converted into radiation in the substrate. We suggest two ways of defining the effective radius of the light source: (i) the half-width at half-maximum of the doughnut shaped spot in the real-space image and (ii) the SPP propagation length (distance $L_{S P P}$ after which SPP intensity has decreased by a factor $\frac{1}{e}$ ). Using the first definition yields a footprint of $\Sigma \approx 0.4 \mu \mathrm{m}^{2}$, which is more than two orders of magnitude lower than for the plasmonic lens; however, this is arbitrary since the size of the doughnut-shaped spot depends on the point spread function of the imaging system and this definition of the source size omits part of the propagating SPPs. The second definition for the source size on the thin gold film leads to $\Sigma \approx 700 \mu \mathrm{m}^{2}\left(L_{S P P} \approx 15 \mu \mathrm{m}\right.$ at $\lambda_{0}=700 \mathrm{~nm}$, see p. 11 of Ref $\left.^{16}\right)$, which is seven times as large as that of the plasmonic lens. Indeed, the tunnel junction formed by the STM tip on the gold film behaves as a nanoscale source of SPPs, which has typical radius ${ }^{17}$ of about $10 \mathrm{~nm}$, but the resulting light source emitting in the substrate is much more extended, due to the propagation over micrometers of the SPPs leaking in the substrate.

For both kinds of sources, the emission pattern may be modeled as a light cone, whose solid angle is $\Omega=$ $2 \pi(1-\cos \theta)$, where $2 \theta$ is the aperture angle of the cone $(\theta$ is the angular beam divergence in our study). Thus, the light emitted in the substrate upon electrical excitation of SPPs is characterized by a solid angle of $\Omega=1.69$ sr for the thin gold film $\left(\theta=43^{\circ}\right)$ and $1.38 \times 10^{-2}$ sr for the plasmonic structure $\left(\theta=4^{\circ}\right)$. Note that the solid angle for the plasmonic lens is more than two orders of magnitude lower than that for the unstructured gold film. In order to compare the spatial and angular beam extents of the two light sources, we may define the étendue of the source $\mathcal{G}$, as $\mathcal{G}=\pi n^{2} \Sigma \sin ^{2} \theta$, where $n$ is the refractive index of the medium (i.e., the glass substrate). Ideally, one could wish for a small, yet collimated source, i.e., a small value of $\mathcal{G}$. However, in general, the smaller the source, the more divergent it is. Optimizing the value of $\mathcal{G}$ may be thought of as a compromise between source size and divergence. For the plasmonic lens we obtain $\mathcal{G} \approx 3.1 \mu \mathrm{m}^{2}$. For the continuous thin gold film, one finds $\mathcal{G} \approx 1.4 \mu \mathrm{m}^{2}$ or $\mathcal{G} \approx 2.4 \times 10^{3} \mu \mathrm{m}^{2}$, depending on whether the source area is defined with regards to the size of the doughnut-shaped spot or the SPP propagation length, respectively. In the case of the doughnut-shaped spot, we see that both the film and the lens have comparable étendues $\mathcal{G}$, whereas using the second definition for the source size for the film, the plasmonic lens clearly surpasses the source based on an unstructured gold film. The aim of the above demonstration is to show that, while the micrometer-sized plasmonic lens is orders of magnitude larger than the nanometer-sized tunnel junction, this structure yields a reduction of the emission solid angle by two orders of magnitude, thus preserving (or improving) the spatio-angular extent of the light source. For comparison purposes, let us consider the electrically driven light nanosource based on the STM-excitation of a triangular, gold nanoparticle on a transparent substrate introduced in Ref. ${ }^{10}$ The edge length $s$ of particle is $130 \mathrm{~nm}$. In this case, the emission is spread over all directions in the substrate, i.e., $\Omega=2 \pi$, the source area is $\Sigma=\frac{\sqrt{3}}{4} s^{2} \approx 7 \times 10^{-3} \mu \mathrm{m}^{2}$ and the étendue $\mathcal{G}$ is $\approx 5 \times 10^{-2} \mu \mathrm{m}^{2}$. This light source relies only on localized surface plasmons and not on propagating SPPs, yielding strong spatial localization of the source but no collimation.

\subsection{Effect of the Plasmonic Lens Parameters}

In Section 3.1, we compared the emission properties of a plasmonic lens and a thin continuous gold film upon electrical excitation using the STM tip. Now we diskuss the effect of varying the geometrical parameters of the plasmonic lens, namely the inner diameter $D$, the number of slits $N$ and the slit period $P$. Here we focus on the dependence of the angular divergence of the emitted light beam on these parameters.

Figure 4(a) shows the variation of the angular divergence versus the inner diameter $D$ for three plasmonic lenses of different silt period $P$. The lowest divergence (i.e., the most efficient beaming) is systematically achieved 
(a)

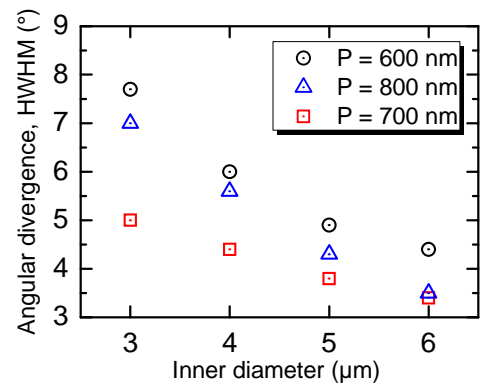

(d)

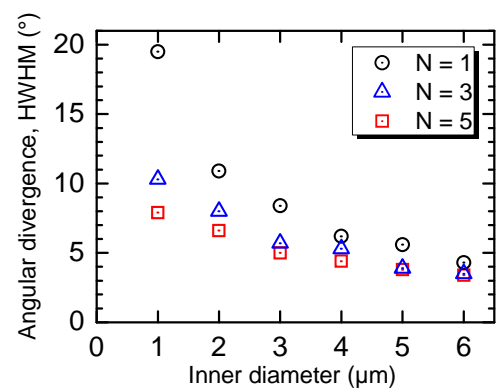

(b)

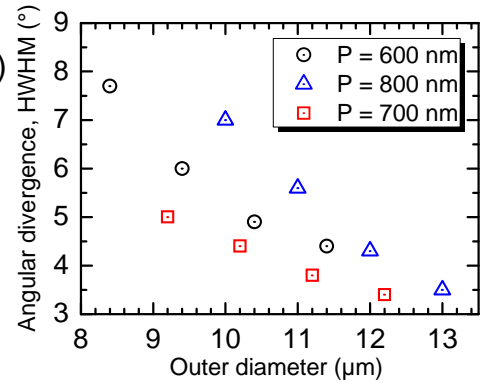

(e)

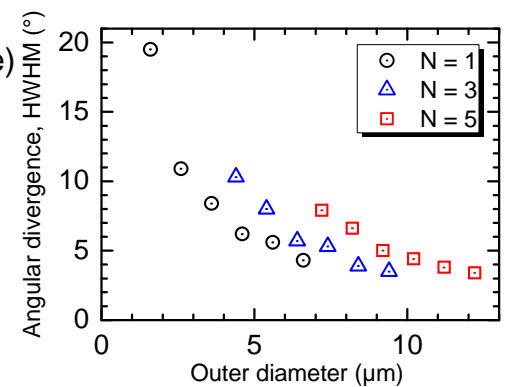

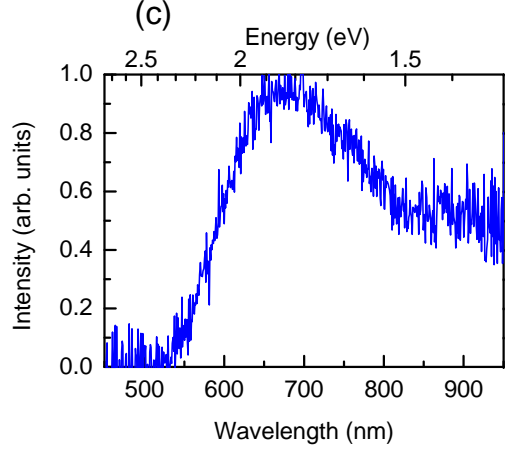

Figure 4. Effect of the geometrical parameters of the plasmonic lenses on the angular beam divergence of their emission. Plasmonic lenses of different inner diameters $D$, slit grating periods $P$ and numbers of slits $N$ are electrically excited with the STM tip located at the central position. Angular beam divergence is retrieved from the half width at half maximum (HWHM) of the doughnut shaped spot in the Fourier-space images and plotted versus either (a,d) the inner diameter $D$ or (b,e) the outer diameter $D^{\prime}=D+2[(N-1) P+W]$ of the plasmonic lens. (c) Emission spectrum, which spreads over the low-energy part of the visible range and the near infrared. The emission is spectrally centered at $\lambda_{0} \approx 700 \mathrm{~nm}$.

for $P=700 \mathrm{~nm}$ as compared to the results with $P=600 \mathrm{~nm}$ and $P=800 \mathrm{~nm}$. This can be understood by considering the emission spectrum shown in Fig. 4(c), which is measured on a thin gold film. This data gives insight into the energy distribution of the surface plasmon nanosource when the tip is made of tunsgten and the sample is made of gold (see also Refs. ${ }^{18,19}$ ). This distribution exhibits a peak centered at about $700 \mathrm{~nm}$ (photon wavelength in vacuum). The beaming effect from our structures is due to the constructive interference of the light emitted from the slits when the slit period equals the SPP wavelength (which is close to the photon wavelength in vacuum); therefore, more of the source energy is converted into a collimated light beam when the energy of maximum emission matches the slit period of the plasmonic lens. The origin of the beamed emission also explains the effect of the number of slits. As shown in Fig. 4(d), the higher the number of slits, the lower the angular divergence of the emitted beam. The slits behave as coherent light sources that interfere in the far field; thus, the most numerous these sources, the sharper the features in the interference pattern. Interestingly, at a fixed inner diameter $D$, increasing $N$ from 1 to 3 strongly enhances the collimation of the emitted beam, whereas adding another two rings $(N=5)$ yields only subtle improvement. This suggests that near-optimal beaming is achieved for $N=3$. Indeed, SPP scattering at the slits decreases their propagation length; as a result, the emission from the slits is comparatively low beyond the third one, as may be seen in Fig. 3(a).

A general feature emerging from Fig. 4 is that the larger the inner diameter, the better the collimation of the emitted beam. The reason is that the $k$-vector distribution of the electric field in the far field (whose squared modulus is the Fourier image) is the Fourier transform of its spatial distribution in the sample plane (whose squared modulus is the real-plane image). As a consequence, larger structures may have narrower angular radiation distributions. To account for this, we plot in Figs. 4(b) and 4(e) the data diskussed above as a function of the outer diameter, which is $D^{\prime}=D+2[(N-1) P+W]$ ( $W$ is the width of the slit). $D^{\prime}$ is the actual size of the light source. This way of plotting the data reveals that a single-ring structure provides an interesting trade-off between the footprint of the source and the angular divergence of the emitted beam, which may be crucial if integration within a device imposes size requirements and if angular divergence is the only criterion 


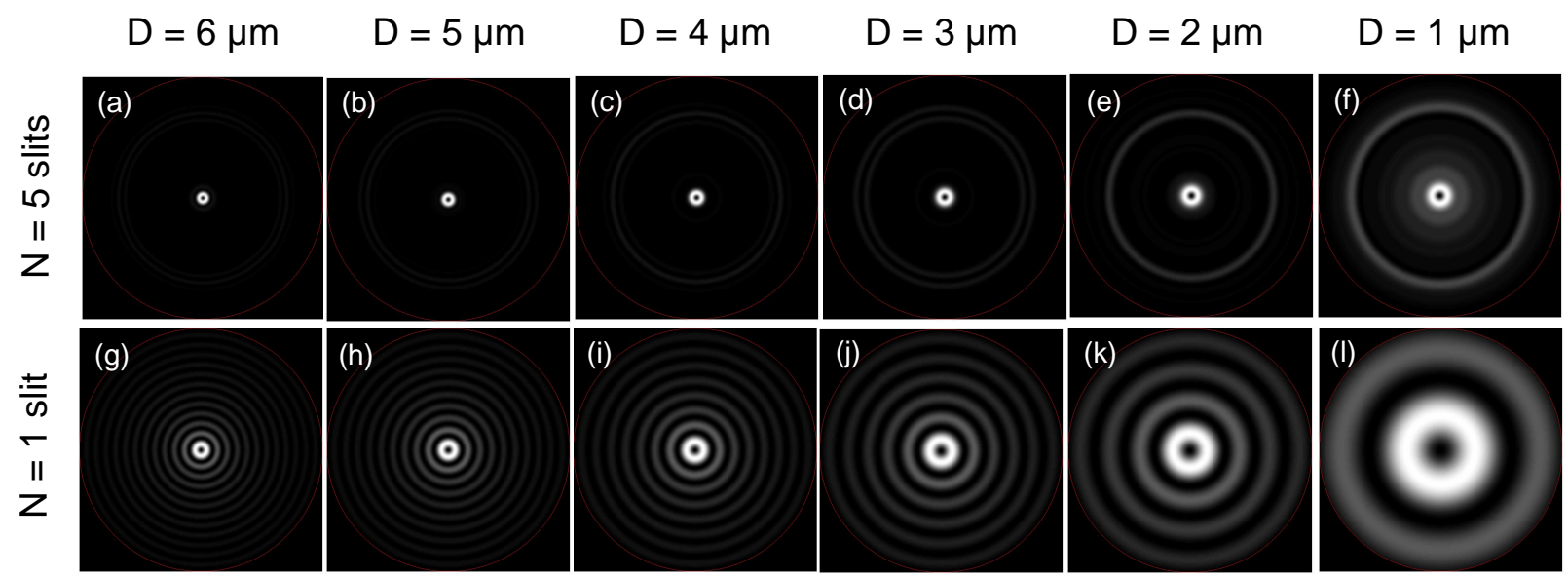

Figure 5. Theoretical Fourier-space images calculated within a monochromatic dipole model with $\lambda_{0}=700 \mathrm{~nm}$ for (a to f) a 5 -slit and (g to l) a single-slit plasmonic lens of inner diameters of 6 to $1 \mu \mathrm{m}$. Excitation at the center of the plasmonic lens is assumed. In panels a to $\mathrm{f}$, the slit grating period is $P=700 \mathrm{~nm}$.

for beam collimation. Indeed, the occurrence of secondary lobes in the angular radiation pattern may have a strong impact on the performance of the light source, depending on the targeted applications. This is why the full Fourier image also must be examined, as diskussed below.

Figure 5 shows calculated Fourier images for plasmonic lenses of inner diameter $D$ varying from 1 to $6 \mu \mathrm{m}$ and consisting of a single slit or a 5 -slit grating of period $P=700 \mathrm{~nm}$. These images have been calculated using a dipole model that is described in Ref. ${ }^{14}$ This series of images confirms that (i) the larger the inner diameter, the lower the angular divergence, (ii) for a given $D$, the angular divergence is much lower with five slits than with one, (iii) the central lobe in the radiation pattern is less sharp with $N=5$ and $D=1 \mu \mathrm{m}$ than with $N=1$ and $D=6 \mu \mathrm{m}$ even though the former has a larger outer diameter. In addition, Fig. 5 reveals the appearance of numerous side lobes around the central lobe at larger emission angles in the case of a single-slit structure. For the five-slit structure, the emission pattern essentially consists of a single lobe. This is due to the destructive interference of the light emitted from the multiple slits in off-axis directions, thus canceling out the side lobes. Hence, if not only low angular divergence is required for a specific application, but also the absence of side lobes, then a 5 -slit lens is to be favored.

Also of importance for potential applications, we have investigated the robustness of the emitted beam collimation and radial polarization against excitation source off-centering. ${ }^{14}$ Although we do not reproduce the full results of this study here, in Fig. 6 we give examples of pronounced off-centered cases, where the effects on the emission properties are obvious. Here we consider a 5-slit grating structure with $D=5 \mu \mathrm{m}$ and $P=700 \mathrm{~nm}$. In Figs. 6(a) to 6(c), SPPs are electrically excited from a location that is $1.2 \mu \mathrm{m}$ away from the center. The theoretical Fourier image reveals an intricate angular distribution of the emission, with several lobes instead of the single doughnut shaped spot observed for tip positions close to center. Theoretical images with a polarization filter also indicate the loss of the radial nature in the emission polarization. In Fig. 6(d), we examine a case where the STM tip is located closer to the inner edge of the first slit than to the center of the structure (tip is off-centered by $1.8 \mu \mathrm{m})$. The experimental image, taken from Ref., ${ }^{14}$ is shown in Fig. 6(e). We see that the more off-centered the tip, the more intricate the angular and polarization distribution of the emitted light becomes.

\section{SUMMARY AND CONCLUSION}

In summary, we have compared the results obtained when a plasmonic lens is electrically excited by the tunnel current from an STM, with those obtained when the STM tip is used to excite SPPs on a thin gold film. While the light emitted from the plasmonic lens is distributed over a larger area $(\approx 10 \mu \mathrm{m})$, the maximum intensity from the thin gold film is concentrated in $\mathrm{a} \approx 1 \mu \mathrm{m}$-diameter region (full-width at half-maximum). The "opposite" 

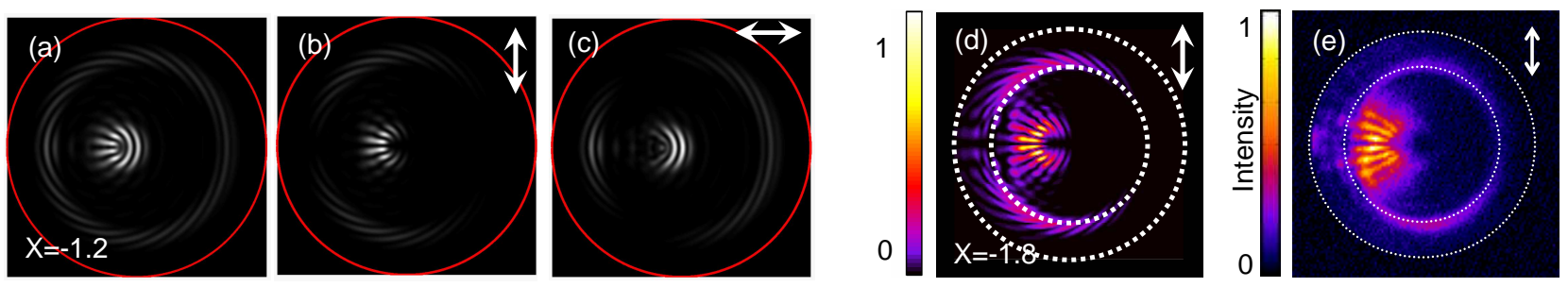

Figure 6. Theoretical Fourier-space images calculated within a monochromatic dipole model with $\lambda_{0}=700 \mathrm{~nm}$ for a 5 -slit plasmonic lens of inner diameter $D=5 \mu \mathrm{m}$ and off-centered excitation at $X=-1.2 \mu \mathrm{m}$ along the $x$ axis of the plasmonic lens $(X=0$ is the center of the structure). (a) intensity image and (b,c) polarization filtered images with the polarizer along (b) the $y$ axis or (c) the $x$ axis. The outer circle (red line) delineates the accessible region of Fourier space. (d) Same as b for $X=-1.8 \mu \mathrm{m}$ and (e) corresponding experimental image. Both images are in false colors and the image in panel e is adapted from Fig. $2 \mathrm{~h}$ of Ref. ${ }^{14}$ Circles (dotted white lines) indicates the critical angle at an air-glass interface and the maximum acceptance angle of the objective lens.

results are obtained for the angular distribution of the emitted light: a low divergence beam (half-width at halfmaximum about $4^{\circ}$ ) is generated with the plasmonic lens, while almost all the light from the thin film is emitted at an angle greater than the air/glass critical angle. This is further quantified by considering the étendues of the two sources: using the definition just above for the size of the source on the gold film, the étendues of the two sources are similar $\left(1.4 \mu \mathrm{m}^{2}\right.$ for the thin film and $3.1 \mu \mathrm{m}^{2}$ for the plasmonic lens). Thus the increase in source size of the plasmonic lens is compensated by the reduction in beam divergence, yielding a similar spatio-angular source extent as compared to the local STM excitation of a thin gold film.

The effect of the geometrical parameters on the plasmonic lens performance were also investigated in this study. It was seen that the lowest divergence beam is obtained when the slit grating period is equal to the wavelength of maximum emission. This is explained by realizing that more of the light will be at the lens' "working wavelength" in this case. Increasing the number of slits decreases the beam divergence, as is typical in a grating structure. Adding more and more slits, however, would not continue improving the beam divergence, since the SPP propagation length is short $(\approx 3 \mu \mathrm{m})$ in the grating. Theoretical calculations using a dipole model show that fewer rings lead to more side lobes in the emission pattern. Such calculations as a function of tip excitation position also demonstrate the very complicated emission pattern that results when the tip is not centered.

In conclusion, we have shown that the local excitation of a plasmonic lens with an STM leads to a radially polarized beam of light of low divergence. In order to optimize the divergence of such a light source, the grating period should match the wavelength of maximum emission of the STM excitation, the lens should consist of at least 3 rings, and the STM excitation must be in the center of the lens. Such electrically excited light sources, together with their radial polarization, have applications in various fields such as particle trapping ${ }^{20}$ and high resolution imaging. ${ }^{21}$

\section{ACKNOWLEDGMENTS}

This work is supported by the Région Ile-de-France in the framework of DIM Nano-K (CNano IdF, COSSMET project). The financial support of China Scholarship Council (CSC) (No. 201304910386) is gratefully acknowledged. The authors thank Thierry Fournier and Jean-François Motte at the NanoFab facility in Grenoble for the fabrication of the plasmonic structures.

\section{REFERENCES}

1. H. Ditlbacher, J. R. Krenn, B. Lamprecht, A. Leitner, and F. R. Aussenegg, "Spectrally coded optical data storage by metal nanoparticles," Opt. Lett. 25, pp. 563-565, Apr 2000.

2. P. Zijlstra, J. W. M. Chon, and M. Gu, "Five-dimensional optical recording mediated by surface plasmons in gold nanorods," Nature 459, pp. 410-413, May 2009. 
3. W. L. Barnes, A. Dereux, and T. W. Ebbesen, "Surface plasmon subwavelength optics," Nature 424, pp. 824-830, Aug. 2003.

4. M. L. Brongersma and V. M. Shalaev, "The case for plasmonics," Science 328(5977), pp. 440-441, 2010.

5. A. V. Zayats and I. I. Smolyaninov, "Near-field photonics: surface plasmon polaritons and localized surface plasmons," J. Opt. A: Pure Appl. Opt. 5(4), p. S16, 2003.

6. T. Wang, E. Boer-Duchemin, Y. Zhang, G. Comtet, and G. Dujardin, "Excitation of propagating surface plasmons with a scanning tunnelling microscope," Nanotechnology 22, p. 175201, Apr. 2011.

7. P. Bharadwaj, A. Bouhelier, and L. Novotny, "Electrical Excitation of Surface Plasmons," Phys. Rev. Lett. 106, p. 226802, June 2011.

8. E. Boer-Duchemin, T. Wang, E. Le Moal, B. Rogez, G. Comtet, and G. Dujardin, "Local low-energy electrical excitation of localized and propagating surface plasmons with a scanning tunneling microscope," in Nanophotonics V, Proc. SPIE 9126, pp. 91260K-91260K-12, May 2014.

9. E. Boer-Duchemin, T. Wang, E. Le Moal, and G. Dujardin, "Electrically driven surface plasmon nanosources," in Ultrafast Phenomena and Nanophotonics XIX, Proc. SPIE 9361, pp. 93610R-93610R11, Feb. 2015.

10. E. Le Moal, S. Marguet, B. Rogez, S. Mukherjee, P. Dos Santos, E. Boer-Duchemin, G. Comtet, and G. Dujardin, "An electrically excited nanoscale light source with active angular control of the emitted light," Nano Lett. 13, pp. 4198-4205, Sept. 2013.

11. T. Wang, B. Rogez, G. Comtet, E. Le Moal, W. Abidi, H. Remita, G. Dujardin, and E. Boer-Duchemin, "Scattering of electrically excited surface plasmon polaritons by gold nanoparticles studied by optical interferometry with a scanning tunneling microscope," Phys. Rev. B 92, p. 045438, July 2015.

12. B. Rogez, R. Horeis, E. Le Moal, J. Christoffers, K. Al-Shamery, G. Dujardin, and E. Boer-Duchemin, "Optical and Electrical Excitation of Hybrid Guided Modes in an Organic NanofiberGold Film System," J. Phys. Chem. C 119, pp. 22217-22224, Sept. 2015.

13. E. Le Moal, S. Marguet, D. Canneson, B. Rogez, E. Boer-Duchemin, G. Dujardin, T. V. Teperik, D.-C. Marinica, and A. G. Borisov, "Engineering the emission of light from a scanning tunneling microscope using the plasmonic modes of a nanoparticle," Phys. Rev. B 93, p. 035418, Jan. 2016.

14. S. Cao, E. Le Moal, E. Boer-Duchemin, G. Dujardin, A. Drezet, and S. Huant, "Cylindrical vector beams of light from an electrically excited plasmonic lens," Appl. Phys. Lett. 105, p. 111103, Sept. 2014.

15. A. Drezet, A. Hohenau, D. Koller, A. Stepanov, H. Ditlbacher, B. Steinberger, F. Aussenegg, A. Leitner, and J. Krenn, "Leakage radiation microscopy of surface plasmon polaritons," Mater. Sci. Eng. B 149, pp. 220-229, Apr. 2008.

16. T. Wang, Excitation électrique de plasmons de surface avec un microscope à effet tunnel. PhD thesis, Université Paris-Sud, 2012.

17. R. W. Rendell, D. J. Scalapino, and B. Mühlschlegel, "Role of local plasmon modes in light emission from small-particle tunnel junctions," Phys. Rev. Lett. 41, pp. 1746-1750, Dec 1978.

18. Y. Zhang, E. Boer-Duchemin, T. Wang, B. Rogez, G. Comtet, E. Le Moal, G. Dujardin, A. Hohenau, C. Gruber, and J. R. Krenn, "Edge scattering of surface plasmons excited by scanning tunneling microscopy," Opt. Express 21, p. 13938, June 2013.

19. T. Wang, G. Comtet, E. Le Moal, G. Dujardin, A. Drezet, S. Huant, and E. Boer-Duchemin, "Temporal coherence of propagating surface plasmons," Opt. Lett. 39, p. 6679, Dec. 2014.

20. Q. Zhan, "Trapping metallic rayleigh particles with radial polarization," Opt. Express 12, pp. 3377-3382, Jul 2004.

21. C. J. R. Sheppard and A. Choudhury, "Annular pupils, radial polarization, and superresolution," Appl. Opt. 43, pp. 4322-4327, Aug 2004. 